\title{
BMJ Open Factors associated with the length of stay at health facilities after childbirth among mothers and newborns in Dhading, Nepal: a cross-sectional study
}

\author{
Subaru lkeda (D) , ${ }^{1}$ Akira Shibanuma (D) , ${ }^{1}$ Ram Silwal, ${ }^{1,2}$ Masamine Jimba (D) ${ }^{1}$
}

To cite: Ikeda S, Shibanuma A, Silwal $\mathrm{R}$, et al. Factors associated with the length of stay at health facilities after childbirth among mothers and newborns in Dhading, Nepal: a cross-sectional study. BMJ Open 2021;11:e042058. doi:10.1136/ bmjopen-2020-042058

- Prepublication history and additional supplemental material for this paper are available online. To view these files, please visit the journal online (http://dx.doi.org/10.1136/ bmjopen-2020-042058).

Received 24 June 2020 Revised 12 March 2021 Accepted 21 March 2021
Check for updates

(c) Author(s) (or their employer(s)) 2021. Re-use permitted under CC BY-NC. No commercial re-use. See rights and permissions. Published by BMJ.

${ }^{1}$ Department of Community and Global Health, Graduate School of Medicine, The University of Tokyo, Tokyo, Japan

${ }^{2}$ Green Tara Nepal, Kathmandu, Nepal

Correspondence to

Dr Akira Shibanuma;

shibanuma@m.u-tokyo.ac.jp

\section{ABSTRACT}

Objective To measure the length of stay at a health facility after childbirth, identify factors associated with the length of stay and measure the gap between the timings of the last check-up and discharge.

Design A cross-sectional study.

Setting Five public health facilities in Dhading, Nepal. Participants 351 randomly selected mothers who gave birth at selected health facilities within 1 year of data collection between 10 and 31 August 2018.

Outcome measure Length of stay (hours) at a health facility after childbirth. Adequate length was defined as 24 hours or longer based on the WHO guidelines.

Results Among 350 mothers (99.7\%) out of 351 recruited, $61.7 \%$ were discharged within 24 hours after childbirth. Factors associated with shorter length of stay were as follows: travel time less than $30 \mathrm{~min}$ to a health facility (incidence rate ratio (IRR) $=0.69,95 \% \mathrm{Cl} 0.61$ to 0.78 ); delivery attended by auxiliary staff (IRR $=0.86$, $95 \% \mathrm{Cl} 0.75$ to 0.98 ); and delivery in a primary healthcare centre (IRR=0.67, 95\% $\mathrm{Cl} 0.58$ to 0.79 ). Factors associated with longer length of stay were as follows: aged 22 years or above at the first pregnancy (IRR=1.25, 95\% Cl 1.13 to 1.40); having maternal complications (IRR $=2.41,95 \% \mathrm{Cl}$ 2.16 to 2.70); accompanied by her own family (IRR=1.17, $95 \% \mathrm{Cl} 1.03$ to 1.34), accompanied by her husband (IRR=1.16, $95 \% \mathrm{Cl} 1.04$ to 1.29 ); and delivered at a facility with a physical space where mother and newborn could stay overnight (IRR=1.20, 95\% $\mathrm{Cl} 1.07$ to 1.34 ). Among mothers without complications, $32 \%$ received the last check-up 3 hours or less before discharge.

Conclusions Multiple factors, such as mothers' conditions, health facility characteristics and external support, were associated with the length of stay after childbirth. However, even if mothers stayed long, they might have not necessarily received timely and proper assessment before discharge.

\section{INTRODUCTION}

Receiving care continuously from pregnancy to the postpartum period is fundamental in improving maternal and neonatal health. ${ }^{1}$ Globally, an estimated $45 \%$ of newborns and $61 \%$ of mothers received postnatal care (PNC) within 2 days after childbirth. An estimated $86 \%$ of pregnant women received at
Strengths and limitations of this study

- Detailed data were collected from mothers on the length of stay at a health facility and its associated factors, particularly on their experiences during pregnancy and childbirth.

- Data from mothers were obtained by visiting their houses to minimise the attrition of mothers listed in the medical records at health facilities.

- Detailed information was obtained from an openended question, which showed reasons behind mothers' choices of the length of stay at health facilities.

- Participants of this study were women who gave birth only at five public health facilities in a rural district of Nepal.

- Mothers' data were collected based on their selfreports in the last 1 year, which may be subjected to recall bias.

least one antenatal care (ANC), and an estimated $76 \%$ gave birth in a health facility in 2013-2018. ${ }^{2}$ The utilisation of PNC remains lower than that of ANC and delivery care by skilled birth attendants. The WHO recommends all mothers and newborns to receive at least four times of PNC: within 24 hours, on day 3 , between days 7 and 14 and 6 weeks after childbirth. The WHO also recommends that mothers stay at a health facility for at least 24 hours after childbirth, as this is the most critical period for the health of both mothers and newborns. ${ }^{1}$ Newborns are recommended to receive check-ups around an hour after birth and before discharge, in addition to an immediate assessment at birth, during the stay. ${ }^{3}$ However, in many low-income and middle-income countries (LMICs), postnatal mothers and newborns are discharged from health facilities too early to receive adequate care. ${ }^{4}$ Early discharge increases the risks of morbidity and mortality in infants owning to insufficient time to detect, diagnose or treat complications. ${ }^{5}$ 
The length of stay at a health facility after childbirth varies across countries. ${ }^{4}$ Among 71 low-income, middleincome and high-income countries, the country-level mean length of stay ranged from 0.5 to 6.2 days for singleton vaginal deliveries. ${ }^{4}$ Owing to a short length of stay, mothers have limited opportunities to attain health education for themselves and their newborns. This can result in reducing mothers' confidence in parenting, increasing breastfeeding problems, maternal depression and dissatisfaction with care ${ }^{67}$ A short length of stay is also associated with an increased risk of readmission. ${ }^{8-10}$ The appropriate length of stay can be the key in improving health outcomes.

According to a prior quantitative study, the following factors were associated with longer length of stay: delivery attended by a physician (reference group as nurse/ midwife, auxiliary staff or others), older maternal age, lower parity, lower economic status or more medical needs such as caesarean section and low birth weight. ${ }^{4}$ In contrast, the following factors were associated with shorter length of stay: younger maternal age, receiving ANC after 3 months of gestation, delivery in public hospitals, mother or relatives' decision to seek a discharge and lack of health workers' insistence on staying longer. ${ }^{11}$ According to a mixed-method study, the following facility-based factors were identified to influence the timing of discharge: (1) insufficient capacity of health service provisions, such as pressure to be discharged because of a limited supply of beds, (2) insufficient capacity for those who accompany with women, such as lack of places to cook or sleep and (3) inappropriate environment so that women could stay long, such as uncleanliness and noise. ${ }^{12}$ Previous quantitative studies investigated factors associated with the length of stay, such as sociodemographic characteristics, the physical condition of a mother and a newborn and reproductive history, which are difficult to modify. Some of the factors found in qualitative studies, however, have not been investigated in quantitative studies. Few studies have investigated the facility environment, health provider characteristics and external support in LMICs.

In most LMICs, the majority of postpartum mothers and newborns do not receive routine care by licenced health personnel in the early postnatal period when the risk of death is the highest owing to a short length of stay at a health facility. ${ }^{21314}$ Even though mothers and newborns are discharged from a health facility within 24 hours after childbirth, which is not recommended by the WHO guidelines, they might be able to receive PNC through home visiting services by health professionals if health resources are sufficient. Mothers may also be able to receive PNC by visiting health facilities by themselves if they have the means to access healthcare. Nevertheless, only $8 \%$ visited health facilities to verify the health of newborns over the first week after childbirth, and 23\%-65\% visited over the first 6 weeks in LMICs in Asia and Africa. ${ }^{13}$ Many studies have examined factors that influence the use of PNC, such as a lower socioeconomic status, living in a rural area and less ANC use. ${ }^{14-16}$ Apart from the characteristics of mothers, many South Asian countries have local practices that confine postpartum mothers and newborns to their home. ${ }^{17}$ Once mothers are discharged from health facilities after childbirth, most do not have an opportunity to receive a check-up by health personnel. While PNC before being discharged from a health facility is primarily for care during the most critical period to prevent mortality, it is the last PNC opportunity if they do not return to a health facility after being discharged or do not receive home visiting services for PNC.

In Nepal, PNC is traditionally uncommon, and most mothers and newborns receive their first PNC after discharge at 6 weeks after childbirth for immunisation. ${ }^{18}$ Ministry of Health defines the timings and contents of PNC; the first PNC should be provided within the first 24 hours of delivery. ${ }^{19}$ The contents of PNC are as follows: (1) identification and management of complications of mothers and newborn and referrals to appropriate health facilities, (2) promotion of exclusive breastfeeding, personal hygiene and nutrition education, and postnatal vitamin $\mathrm{A}$ and iron supplementation for mothers, (3) immunization for newborns, and (4) family planning counselling and services. ${ }^{19}$ Although $58 \%$ of mothers and $57 \%$ of newborns received at least one PNC within 48 hours after childbirth in 2016, this coverage also included mothers and newborns who were checked and discharged immediately after childbirth. ${ }^{19}$ Among mothers who gave birth in a health facility, $47 \%$ were discharged before the recommended 24 hours, and it is unknown whether they received PNC at the recommended timing and whether they received all the recommended three times of PNC after discharge. ${ }^{20}$ Moreover, a disparity exists in the coverage of PNC between rural and urban areas in Nepal. The utilisation of PNC within 2 days after childbirth in urban areas was 1.6 times higher than that in rural areas in 2014. ${ }^{21}$ Especially in rural or mountainous areas, where health resources and access are limited, mothers and newborns can receive PNC after birth and within 24 hours of delivery by staying long enough in a health facility after childbirth. Thus, identifying modifiable factors in resource-limited settings will be a key to developing a community-level intervention for mothers and newborns to stay long enough in a health facility and to receive routine PNC.

This study was conducted: (1) to measure the length of stay at a health facility after childbirth, (2) to identify the factors associated with the length of stay at a health facility after childbirth and (3) to measure the gap between the timings of the last check-up and discharge in Dhading, Nepal.

\section{METHODS}

\section{Study settings}

The current study is a community-based cross-sectional study design conducted in Dhading district, Nepal, in 2018. It covers an area of $1926 \mathrm{~km}^{2}$, and $95 \%$ of the land was used for agriculture in 2013. Its population 
was 336067 , and $88 \%$ of them resided in rural areas in 2013. The adult literacy rate among women was $56 \%{ }^{22} 23$ Dhading district is located adjacent to Kathmandu, with the main road running through the southern part of the district connecting Kathmandu and Pokhara. The Dhading district has 56 public health facilities. In each district, primary-level public facilities are health posts, followed by primary healthcare centres (PHCCs) and district hospitals. Both health posts and PHCCs provide basic emergency obstetric and newborn care. Neither of them performs surgeries. Licenced health workers in health posts are auxiliary staff. Moreover, physicians, nurses and laboratory technicians are available at PHCCs. The district hospital provides comprehensive emergency obstetric and newborn care, where surgical treatments are available. A radiologist is available in district hospitals.

The Government of Nepal encourages mothers to receive care at health facilities by providing mothers free maternity care services and incentives when mothers complete four times or more ANC (approximately US\$3.6) and receive delivery care at health facilities (approximately US\$4.5-13.4). Also, health facilities can receive approximately US\$8.9-13.4 for every normal delivery, and health workers can receive approximately US\$2.7 for any delivery. However, such incentives are not provided for PNC. ${ }^{18}$ In the study area, each municipality has nine female community health volunteers (FCHVs) who are local women working as health volunteers. FCHVs encourage mothers to use maternity services and distribute iron tablets. ${ }^{18}$

\section{Eligible participants}

Participants of this study were mothers who gave birth in selected facilities within 1 year of the data collection date. Specifically, the inclusion criteria were: (1) gave birth in selected facilities between August 2017 and July 2018 , (2) lived in the selected area at the date of the interview and (3) aged between 15 and 45 years at the data collection date. The following mothers were excluded: (1) those whose enumerators could not access owing to a geographic reason, (2) who were not able to understand and answer the questions in Nepalese and (3) who could not be identified from the information in medical records.

\section{Sample selection}

Among the 50 public health facilities handling deliveries in the district, five facilities were selected where the highest number of deliveries were reported between July 2016 and July 2017: one district hospital (hospital A), one PHCC (PHCC B) and three health posts (health posts $\mathrm{C}, \mathrm{D}$ and $\mathrm{E}$ ). Mothers were stratified by the level of health facilities. To reflect the responses from mothers who gave birth at health posts, the number of recruited mothers was balanced among the three levels of health facilities. For health posts, the number of mothers was allocated proportionately based on the number of deliveries. Data on the number of deliveries in each facility were obtained from Dhading District Health Office. The lists of mothers were obtained from the selected facilities prior to data collection. Based on the lists, mothers who met the criteria were recruited using simple random sampling. Mothers were identified based on their name, address, age and date of giving birth on the list. Based on the lists of mothers, research assistants visited the selected mothers at their homes or health facilities when they came in for routine immunisation. Mothers who could not be identified were replaced with mothers who lived in the same ward in the lists.

The minimum sample size was calculated using OpenEpi V.3.01, for $80 \%$ as the power, with a $95 \%$ CI, $53 \%$ of unexposed with the outcome and 0.43 as the OR. ${ }^{12}$ The non-response rate was assumed to be $20 \%$. The calculated sample size was approximately 350. A total of 110 mothers were recruited from the hospital, 110 from the PHCC and 130 from the health posts (32, 60 and 38 from health posts $\mathrm{C}, \mathrm{D}$ and $\mathrm{E}$, respectively).

\section{Data collection}

A semistructured questionnaire was developed (available in online supplemental material 1) based on a previous study. ${ }^{12}$ It was initially prepared in English and translated into Nepalese, and then translated back into English to ensure that the translation was accurate. It included four categories: (1) sociodemographic characteristics, (2) reproductive history and information of the latest delivery, (3) characteristics of a health facility and (4) characteristics of external support. The questionnaires also included an open-ended question about reasons for deciding the timing of discharge from a health facility. KoBo Toolbox (https://www.kobotoolbox.org) was used to develop the questionnaire and enter responses from mothers by smartphones.

The questionnaire was pretested prior to the main survey to correct for errors and add suggestive points before finalising the questionnaires. Twenty mothers were selected for pretesting. They were excluded from the main survey. The questionnaire was checked for item difficulty and cultural acceptability by mothers. It was modified based on feedback from respondents of the pretesting. Data collection for the main survey was conducted from 10 to 30 August 2018. Four Nepalese research assistants with a public health background and experience as an enumerator of surveys were recruited for this study. They did not work in the selected facilities. Before the data collection period started, the assistants were trained to acquire interview techniques and to understand the research and ethical considerations. Face-to-face interviews were conducted at each mother's house or at health facilities. After obtaining informed consent from mothers, a research assistant conducted an interview using the questionnaire, which took $30 \mathrm{~min}$ on average.

\section{Variables}

The dependent variable was the length of stay (hours) in a health facility after childbirth, which was based on mothers' 
self-reports. This study defined an adequate length of stay as 24 hours or longer after childbirth, which complied with the WHO guidelines. ${ }^{1}$ The following independent variables were included based on previous studies: education, ethnicity/caste, religion, wealth index, travel time to a health facility, complications during labour, delivery and early postpartum period, age at the first pregnancy, child's birth weight, time of giving birth, having an experience of a child death, birth attendant, level of health facility (hospital/PHCC/health post), having a physical space a mother and a newborn can stay overnight in a facility, components of PNC, FCHVs' support, family members accompanying to a health facility when labour started and husband's share of childcare. ${ }^{12} 19$

FCHV's support was measured by asking whether FCHVs did the following for mothers, as categorised into three levels: (1) advised about the preparedness of the birth, (2) advised to receive ANC in a health facility, (3) advised to receive delivery care in a health facility and (4) visited after childbirth. Furthermore, the components of PNC were measured by asking the following items and categorised into four levels: (1) advised to breastfeed continuously for 6 months without giving the infant other foods and liquids, (2) advised to receive PNC, (3) advised to get nutritious food for the mother's health, (4) explained family planning methods, (5) told how to involve a partner in the issues of family planning, (6) told continuing breastfeeding will extend a period of amenorrhoea, (7) told how to identify the danger signs of postdelivery women, (8) told how to identify the danger signs of newborns, (9) provided vitamin A supplements, (10) provided iron tablets, (11) told to stay with the newborn until discharge from the health facility, (12) told to eat and drink regularly, (13) told to go to the toilet often and (14) provided help during the initiation of breastfeeding of the newborn.

\section{Data analysis}

Negative binomial regression model was used to examine the factors associated with the length of stay, adjusting for other independent variables listed in the previous subsection. It was selected as the dependent variable was a non-negative integer. Moreover, the responses to the open-ended question about the timing of discharge were categorised. Then, the frequencies of each category were tabulated, stratified by the length of stay. Multicollinearity was assessed using variance inflation factors. An automated variable selection method was not used to ensure that the selected independent variables worked as potential confounders in the regression model. To reflect the fact that different percentages of women were sampled across the five facilities, the regression model incorporated a sampling weight calculated based on the percentage of sampled women among the total number of delivered women at a facility.

Incident rate ratio (IRR) was used to examine the sign and size of the association between the independent and dependent variables. The wealth index was derived from the principal components analysis (PCA) of household assets to produce quintile scores. To balance each category, the second and third categories were combined, and the wealth index had a total of three categories. The components of PNC and FCHV support were also derived from PCA and categorised into three and four groups by quintile scores, respectively. For the significance testing, a $\mathrm{p}$ value of less than 0.05 was considered statistically significant. In the negative binomial regression model, the statistical significance was tested to explore if each of the independent variables was associated with the dependent variable; that is, the joint significance of two or more independent variables were not examined. This is why adjustment for multiple comparisons, such as Bonferroni correction, was not made. ${ }^{22}{ }^{23}$ Data were analysed using STATA V.12.

\section{Ethical considerations}

Permission to collect data for the current study was also gained from local authorities. Participation was voluntary, and the confidentiality and anonymity of all data were assured. Informed consent was obtained prior to participation. For women aged between 15 and 18 years at the data collection date, permission was obtained from their guardian.

\section{Patient and public involvement}

The research questions were developed through discussions with staff from a local non-governmental organization and health facilities in Nepal. Study participants were not involved in the design of this study because it may have affected the results of the study. Instead, this study was designed based on the information obtained from local mothers' meetings and interviews with local pregnant women, mothers with small children and their family members who lived in the similar settings. The discussion, meetings and interviews were conducted in August 2017 and March 2018.

\section{RESULTS}

Out of 779 mothers who met the criteria, 351 mothers were randomly selected, though one mother refused to participate. Finally, 350 mothers $(99.7 \%)$ of the 351 recruited mothers were included in the analysis. No missing data were found. According to table 1, 61.7\% $(\mathrm{n}=216)$ were discharged before 24 hours after childbirth, which did not comply with the recommendation by the WHO and the Government of Nepal. The median length of stay was 16 hours (IQR: 10-24). Figure 1 shows the distribution of the length of stay by the health facility. The median length of stay was extensively different among health facilities. PHCC B was more likely to discharge mothers earlier than hospital A and health posts $\mathrm{C}$ and D. Between all cases and uncomplicated cases in figure 1, major differences were seen in hospital A and PHCC B. In these health facilities, the ranges of the length of stay were wider in the bars for all cases (the left figure) than 
Table 1 Characteristics of mothers $(n=350)$

\begin{tabular}{|c|c|c|}
\hline Variables & $\mathbf{N}$ & $\%$ \\
\hline \multicolumn{3}{|l|}{ Length of stay (hours) } \\
\hline$<24$ & 216 & 61.7 \\
\hline$\geq 24$ & 134 & 38.3 \\
\hline \multicolumn{3}{|l|}{ Age of the latest child } \\
\hline $1-4$ month(s) & 78 & 22.3 \\
\hline 5-8 months & 133 & 38.0 \\
\hline 9-12 months & 139 & 39.7 \\
\hline \multicolumn{3}{|l|}{ Education } \\
\hline No formal education & 59 & 16.9 \\
\hline Up to secondary (grades $1-10$ ) & 213 & 60.9 \\
\hline $\begin{array}{l}\text { More than secondary (grade } 11 \text { or } \\
\text { above) }\end{array}$ & 78 & 22.3 \\
\hline \multicolumn{3}{|l|}{ Ethnicity/caste } \\
\hline Brahmin/Chhetri & 118 & 33.7 \\
\hline Dalit & 41 & 11.7 \\
\hline Janajati & 188 & 53.7 \\
\hline Other & 3 & 0.9 \\
\hline \multicolumn{3}{|l|}{ Religion } \\
\hline Hindu & 310 & 88.6 \\
\hline Buddhist & 22 & 6.3 \\
\hline Others & 18 & 5.1 \\
\hline \multicolumn{3}{|l|}{ Wealth index } \\
\hline Poorest & 160 & 42.3 \\
\hline Middle & 146 & 46.4 \\
\hline Richest & 44 & 11.4 \\
\hline \multicolumn{3}{|l|}{ Travel time to a health facility (min) } \\
\hline$<30$ & 200 & 57.1 \\
\hline $30-59$ & 70 & 20.0 \\
\hline $60-119$ & 48 & 13.7 \\
\hline $120-240$ & 32 & 9.1 \\
\hline
\end{tabular}

Complication during labour, delivery and early postpartum period

$\begin{array}{lrr}\text { Yes } & 61 & 17.4 \\ \text { No } & 289 & 82.6\end{array}$

Age at the first pregnancy (range 15-34; median=20) (years)

$\begin{array}{lrr}<18 & 38 & 10.9 \\ 18-21 & 239 & 68.3 \\ \geq 22 & 73 & 20.9\end{array}$

Living with children aged under 5 years except the latest child

$\begin{array}{lll}\text { Yes } & 287 & 82.0 \\ \text { No } & 63 & 18.0\end{array}$

Child's birth weight $(\mathrm{kg})$

$\begin{array}{lrr}<2.5 & 80 & 22.8 \\ 2.5-3.9 & 253 & 72.3 \\ \geq 4 & 17 & 4.9\end{array}$

Time of giving birth

Continued

\begin{tabular}{ccc}
\hline Table 1 Continued & & \\
\hline Variables & N & $\%$ \\
\hline 03:00-10:00 & 115 & 32.9 \\
11:00-19:00 & 153 & 43.7 \\
20:00-02:00 & 82 & 23.4 \\
\hline
\end{tabular}

Having experience of a child death, who was born alive but later died

\begin{tabular}{lrr}
\hline Yes & 12 & 3.4 \\
\hline No & 338 & 96.6 \\
\hline Birth attendant & & \\
\hline $\begin{array}{l}\text { Physician } \\
\text { Nurse }\end{array}$ & 143 & 20.3 \\
\hline $\begin{array}{l}\text { Health assistant/auxiliary health worker/ } \\
\text { auxiliary nurse and midwife }\end{array}$ & 136 & 38.9 \\
\hline Health facility a mother gave birth & & \\
\hline District hospital A & 110 & 31.4 \\
\hline Primary healthcare centre B & 110 & 31.4 \\
\hline Health post C & 38 & 10.9 \\
\hline Health post D & 60 & 17.1 \\
\hline Health post E & 32 & 9.14 \\
\hline
\end{tabular}

Having a physical space mothers and newborns could stay overnight in a health facility

$\begin{array}{rrr}\text { Yes } & 280 & 80.0 \\ \text { No } & 70 & 20.0\end{array}$

Components of PNC

\begin{tabular}{lrr} 
Lowest & 85 & 24.2 \\
\hline Lower & 92 & 26.3 \\
Higher & 90 & 25.7 \\
Highest & 83 & 23.7 \\
\hline FCHV's support & & \\
Lower & 193 & 55.1 \\
Middle & 98 & 28.0 \\
Higher & 59 & 16.9
\end{tabular}

Family members accompanied with a mother when labour started to a health facility

\begin{tabular}{lrr} 
Respondent's own family & 36 & 10.3 \\
Family-in-law & 204 & 58.3 \\
Both families & 29 & 8.3 \\
Husband/friend/aunt/alone & 81 & 23.1 \\
Husband's sharing of childcare & & \\
$<40 \%$ & 85 & 24.2 \\
$40 \%-49 \%$ & 97 & 27.7 \\
$50 \%$ or more & 168 & 48.0 \\
\hline
\end{tabular}

FCHV, female community health volunteer; PNC, postnatal care.

for uncomplicated cases (the center figure). Of all, only one mother gave birth by caesarean section.

Table 2 shows the results of the regression analysis. Compared with received education of up to secondary (grades 1-10), more than secondary (grade 11 or above) 


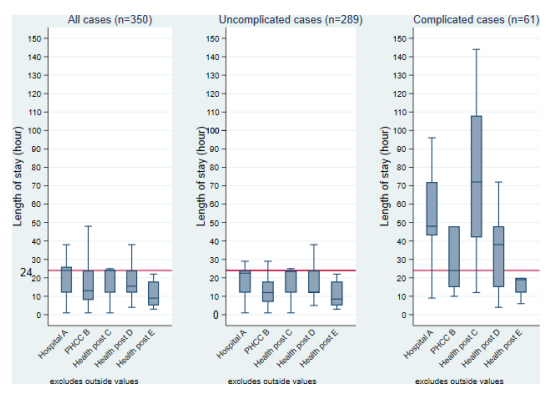

Figure 1 The length of stay after childbirth by health facility (hour). Note: the left figure describes all the mothers $(n=350)$. Mothers who answered that they did not have some types of complications during labour, delivery and early postpartum period were summarised in the centre figure $(n=289)$. Mothers who answered that they had some types of complications were summarised in the right figure $(n=61)$. The red line shows 24 hours as the WHO recommends that mothers and newborns stay at a health facility at least 24 hours after childbirth. ${ }^{1}$ PHCC, primary healthcare centre.

was associated with longer length of stay (IRR $=1.12$, $95 \% \mathrm{CI} 1.01$ to 1.25 ), and received no formal education was associated with shorter length of stay (IRR $=0.87$, $95 \%$ CI 0.77 to 0.99 ). Travel time to a health facility was associated with longer length of stay $(<30 \mathrm{~min}$ : IRR $=0.69$, $95 \%$ CI 0.61 to $0.78 ; 30-59 \mathrm{~min}$ : IRR $=0.85,95 \%$ CI 0.74 to $0.98 ; 120-240 \mathrm{~min}:$ IRR $=0.88,95 \%$ CI 0.72 to 1.09 ; $60-119 \mathrm{~min}$ as the reference group).

Having some types of maternal complications during labour, delivery or early postnatal period was associated with longer length of stay (IRR=2.41, 95\% CI 2.16 to 2.70 ). Aged 22 years or elder at the first pregnancy was associated with longer length of stay (IRR=1.25, 95\% CI 1.13 to 1.40), compared with those aged between 18 and 21 years. Compared with normal birth weight $(2.5-3.9 \mathrm{~kg})$, low birth weight was associated with shorter length of stay (IRR $=0.78,95 \%$ CI 0.71 to 0.87 ), although heavy birth weight had no significant association (IRR $=0.86,95 \% \mathrm{CI}$ 0.72 to 1.04$)$. Mothers who gave birth between 11:00 and 02:00 were more likely to stay longer (11:00-19:00: $\mathrm{IRR}=1.11,95 \%$ CI 1.01 to $1.22,20: 00-02: 00: \mathrm{IRR}=1.15$, $95 \%$ CI 1.02 to 1.30 ), as compared with those who gave birth between 03:00 and 10:00. Having experienced a child's death had no significant association (IRR $=1.15$, $95 \%$ CI 0.93 to 1.42 ).

Delivery attended by an auxiliary staff was associated with shorter length of stay, as compared with delivery attended by a physician and a nurse even after adjusting for complications and other factors $(\mathrm{IRR}=0.86,95 \% \mathrm{CI}$ 0.75 to 0.98 ). Giving birth in the PHCC was associated with shorter length of stay (IRR=0.67, 95\% CI 0.58 to $0.79)$, compared with the health posts. Having physical space to stay overnight in a health facility was associated with longer length of stay (IRR=1.20,95\% CI 1.07 to 1.34 ).

Mothers who had more support from FCHVs were more likely to stay shorter (middle: IRR $=0.84,95 \%$ CI 0.76 to 0.93 , higher: IRR $=0.81,95 \%$ CI 0.72 to 0.92 ), compared with those with less support. Compared with mothers who
Table 2 Negative binomial regression: factors associated with the length of stay $(n=350)$

\begin{tabular}{|c|c|c|c|}
\hline Variables & IRR & $95 \% \mathrm{Cl}$ & $P$ value \\
\hline \multicolumn{4}{|l|}{ Education } \\
\hline $\begin{array}{l}\text { Up to secondary (grades } \\
1-10)\end{array}$ & Reference & & \\
\hline No formal education & 0.87 & 0.77 to 0.99 & 0.030 \\
\hline $\begin{array}{l}\text { More than secondary } \\
\text { (grade } 11 \text { or above) }\end{array}$ & 1.12 & 1.01 to 1.25 & 0.031 \\
\hline \multicolumn{4}{|l|}{ Ethnicity/caste } \\
\hline Janajati & Reference & & \\
\hline Brahmin/Chhetri & 1.02 & 0.92 to 1.13 & 0.727 \\
\hline Dalit & 1.06 & 0.92 to 1.23 & 0.393 \\
\hline Other & 0.73 & 0.45 to 1.18 & 0.198 \\
\hline \multicolumn{4}{|l|}{ Religion } \\
\hline Hindu & Reference & & \\
\hline Buddhist & 0.82 & 0.70 to 0.98 & 0.025 \\
\hline Others & 0.87 & 0.72 to 1.04 & 0.119 \\
\hline \multicolumn{4}{|l|}{ Wealth index } \\
\hline Poorest & Reference & & \\
\hline Middle & 1.02 & 0.91 to 0.13 & 0.767 \\
\hline Richest & 1.10 & 0.94 to 1.29 & 0.246 \\
\hline \multicolumn{4}{|c|}{ Travel time to a health facility (min) } \\
\hline $60-119$ & Reference & & \\
\hline$<30$ & 0.69 & 0.61 to 0.78 & $<0.001$ \\
\hline $30-59$ & 0.85 & 0.74 to 0.98 & 0.022 \\
\hline $120-240$ & 0.88 & 0.72 to 1.09 & 0.239 \\
\hline $\begin{array}{l}\text { Complications during } \\
\text { labour, delivery and soon } \\
\text { after delivery }\end{array}$ & 2.41 & 2.16 to 2.70 & $<0.001$ \\
\hline
\end{tabular}

Age at the first pregnancy (years)

\begin{tabular}{|c|c|c|c|}
\hline $18-21$ & Reference & & \\
\hline$<18$ & 0.95 & 0.82 to 1.10 & 0.515 \\
\hline$\geq 22$ & 1.25 & 1.13 to 1.40 & $<0.001$ \\
\hline \multicolumn{4}{|l|}{ Child's birth weight (kg) } \\
\hline $2.5-3.9$ & Reference & & \\
\hline$<2.5$ & 0.78 & 0.71 to 0.87 & $<0.001$ \\
\hline$\geq 4.0$ & 0.86 & 0.72 to 1.04 & 0.131 \\
\hline \multicolumn{4}{|l|}{ Timing of giving birth } \\
\hline 03:00-10:00 & Reference & & \\
\hline 11:00-19:00 & 1.11 & 1.01 to 1.22 & 0.036 \\
\hline 20:00-02:00 & 1.15 & 1.02 to 1.30 & 0.019 \\
\hline $\begin{array}{l}\text { Having an experience of a } \\
\text { child death, who was born } \\
\text { alive but later died }\end{array}$ & 1.15 & 0.93 to 1.42 & 0.198 \\
\hline \multicolumn{4}{|l|}{ Birth attendant } \\
\hline Nurse & Reference & & \\
\hline Physician & 1.07 & 0.95 to 1.20 & 0.244 \\
\hline $\begin{array}{l}\text { Health assistant/auxiliary } \\
\text { health worker/auxiliary } \\
\text { nurse and midwife }\end{array}$ & 0.86 & 0.75 to 0.98 & 0.027 \\
\hline Level of facility & & & \\
\hline
\end{tabular}

Continued 
Table 2 Continued

\begin{tabular}{|c|c|c|c|}
\hline Variables & IRR & $95 \% \mathrm{Cl}$ & $P$ value \\
\hline Health post & Reference & & \\
\hline $\begin{array}{l}\text { Primary healthcare } \\
\text { centre }\end{array}$ & 0.67 & 0.58 to 0.79 & $<0.001$ \\
\hline District hospital & 0.98 & 0.85 to 1.14 & 0.830 \\
\hline $\begin{array}{l}\text { Having a physical space } \\
\text { mothers and newborns } \\
\text { could stay overnight in a } \\
\text { health facility }\end{array}$ & 1.20 & 1.07 to 1.34 & 0.002 \\
\hline \multicolumn{4}{|l|}{ Component of PNC } \\
\hline Lowest & Reference & & \\
\hline Lower & 0.99 & 0.88 to 1.12 & 0.906 \\
\hline Higher & 0.91 & 0.80 to 1.03 & 0.137 \\
\hline Highest & 1.04 & 0.92 to 1.18 & 0.551 \\
\hline \multicolumn{4}{|l|}{ FCHV's support } \\
\hline Lower & Reference & & \\
\hline Middle & 0.84 & 0.76 to 0.93 & 0.001 \\
\hline Higher & 0.81 & 0.72 to 0.92 & 0.001 \\
\hline \multicolumn{4}{|c|}{$\begin{array}{l}\text { Family members accompanied with a mother when labour started } \\
\text { to a health facility }\end{array}$} \\
\hline Family-in-law & Reference & & \\
\hline $\begin{array}{l}\text { Husband/friend/aunt/ } \\
\text { alone }\end{array}$ & 1.16 & 1.04 to 1.29 & 0.007 \\
\hline Respondent's own family & 1.17 & 1.03 to 1.34 & 0.018 \\
\hline Both families & 1.10 & 0.95 to 1.28 & 0.195 \\
\hline \multicolumn{4}{|c|}{ Husband's sharing of childcare } \\
\hline 50 or more & Reference & & \\
\hline$<40 \%$ & 1.02 & 0.92 to 1.14 & 0.667 \\
\hline $40 \%-49 \%$ & 1.10 & 0.99 to 1.22 & 0.090 \\
\hline
\end{tabular}

$P$ values less than 0.05 are highlighted in a bold font. FCHV, female community health volunteer; IRR, incident rate ratio; PNC, postnatal care.

were accompanied by family-in-laws only, mothers accompanied by their parents, siblings or husband only were more likely to stay longer (respondent's family: IRR $=1.17$, $95 \%$ CI 1.03 to 1.34 ; others: IRR $=1.16,95 \%$ CI 1.04 to 1.29). The husband's share of childcare had no significant association $(<40 \%$ : IRR $=1.02,95 \%$ CI 0.92 to 1.14 ; $40 \%-49 \%$ : IRR $=1.10,95 \%$ CI 0.99 to $1.22 ; 50 \%$ or more as the reference group).

Figure 2 shows the time gaps between the last check-up in the health facility and discharge for mothers without complications and having received check-up before discharge $(n=141)$. Among 141 mothers who did not have complications, $45(31.9 \%)$ received the last check-up 3 hours or less before discharge.

Table 3 summarises the 350 responses from an openended question on reasons for deciding the timing of discharge from a health facility. The responses were divided into the following six categories: physical condition $(n=270)$, health staff's suggestion $(n=129)$, access to the facility $(n=94)$, characteristics of the facility $(n=90)$, family matters $(n=54)$ and the timing of delivery $(n=7)$. Among mothers who mentioned that their delivery was normal as a reason for deciding the timing of discharge $(\mathrm{n}=224)$, about two-thirds $(\mathrm{n}=149)$ stayed less than 24 hours. Among mothers who answered that health personnel advised them to be discharged $(n=129)$, almost half $(n=65)$ stayed less than 24 hours. Mothers who raised both living nearby and far from the health facility as reasons for deciding the timing of discharge were more likely to answer that they stayed less than 24 hours.

\section{DISCUSSION}

This study investigated the situation and factors behind early discharge from a health facility after childbirth in Dhading, Nepal. This study has three major findings. First, after childbirth, more than half of mothers were discharged from a health facility without staying at least 24 hours as recommended, and the length of stay was extensively different across health facilities that mothers gave birth. Second, the following points were associated with the length of stay: (1) characteristics of a health facility, (2) mother's characteristics and (3) external support. Lastly, among mothers without complications, only about one-thirds received the last check-up 3 hours or less before discharge.

In this study, the length of stay varied substantially among health facilities. The distribution of the length of stay by health facility changed when compared with all the cases and uncomplicated cases in hospital A and PHCC B. Mothers with complications might choose these higher-level health facilities and possibly stayed longer. The share of discharge within 24 hours was higher in the study area $(61.7 \%)$ than in the national representative data $(46.5 \%) .{ }^{20}$ Mothers who had complications and who had planned caesarean section may seek higher health facilities in Kathmandu. The markedly low caesarean section rate $(0.3 \%)$ in this study supports this possibility.

Compared with giving birth in the health posts, giving birth in the PHCC were associated with shorter length of stay. This finding contradicted a previous study in Tanzania, which found that delivery in the higher tier of a facility, such as a hospital, was associated with longer length of stay. ${ }^{12}$ Health facility characteristics can explain this contradiction. In addition to the physical condition of mothers and newborns and the health facility's policy related to the timing of discharge, comfortable environment such as temperature, bed and food availability and smell were raised as a reason to leave early in the openended question in this study. Such an environmental situation is commonly seen in many low-income countries. ${ }^{24}$ When the facility had a physical space where a mother and a newborn could stay overnight, mothers were more likely to stay longer in this study. A study in Tanzania shows a reason for leaving health facilities early, such as pressure from a health facility to leave early to accommodate other mothers, facility limitations such as lack of space to stay, cook and do laundry and health facility's 


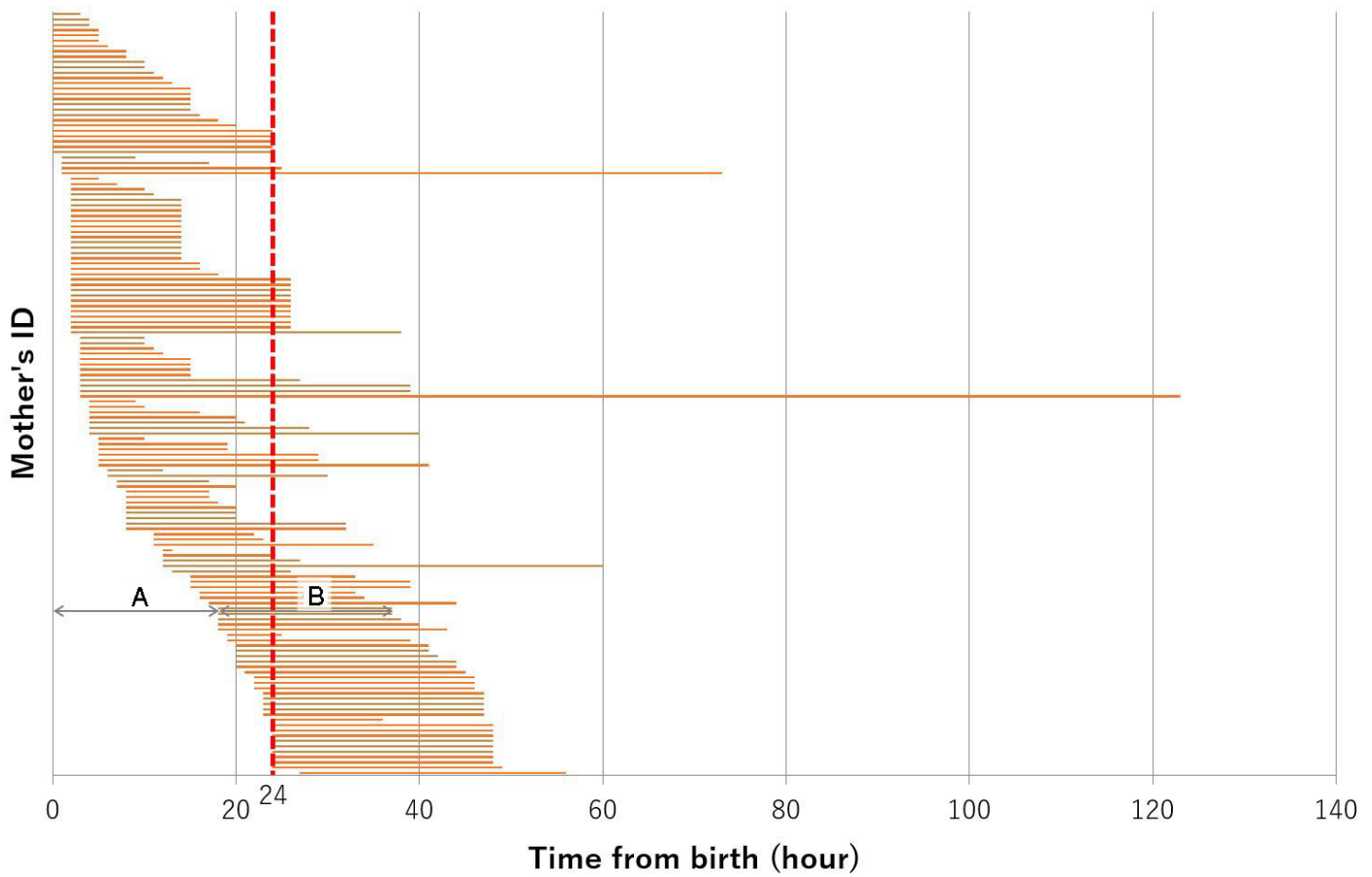

Figure 2 Time gap between timing of the last check-up and discharge $(n=141)$. Figure part A represents time (hours) between childbirth and the last check-up that a mother received before discharge. Figure part B (a red horizontal bar) represents time (hours) between the last check-up and discharge. The following mothers were excluded from this figure: (1) who had some types of complication during labour, delivery or early postnatal period and (2) who did not receive postnatal check during the stay in a health facility or who did not remember whether she received the check.

operation hours. ${ }^{12}$ The results of this study add evidence to these previous findings.

Travel time to a health facility of less than 60 minutes was associated with longer length of stay, as compared with those of $60-119 \mathrm{~min}$. However, no significant difference was seen between mothers whose travel times were $60-119$ and 120-240 min. Mothers who travelled for 120-240 min did not have the option of transportation means other than a stretcher carried by families or neighbours. Thus, some mothers were discharged earlier to fit their family's schedule, as described in the open-ended question. Moreover, as many people engage in agriculture, or raise livestock, it may also be challenging for families to leave their home for a long time to care for their mothers. ${ }^{25}$ In this study, mothers who gave birth between 11:00 and 02:00 were more likely to stay longer as compared with the other period of the day. This is possibly because of poor infrastructure such as road conditions and transportation. ${ }^{26}$ In the open-ended question, mothers raised transportation availability and darkness as a reason for leaving the health facility (table 3 ). After getting dark, going home with a postnatal mother and child without transportation can be difficult in such settings.

Aged 22 years or elder at the first pregnancy was associated with longer length of stay, as compared with younger age at the first pregnancy. This is possibly because with age, they have more knowledge and can gain autonomy in decision making on the use of maternity care services. ${ }^{27}$ Studies in Nepal, India and other countries show that ages at the first birth and marriage are positively associated with the utilisation of ANC..$^{28}$ It can also be applied for the length of stay and PNC use, too. The normal birth weight of a newborn was associated with a longer length of stay in this study. In a previous study, lower birth weight was associated with longer length of stay of mothers. ${ }^{4}$ In this study, 'the length of stay' was defined as the length of stay at the facility where the mother gave birth, and it did not include the time after moving to seek care in a higher level health facility. In low birth weight newborns' cases, mothers might need to leave quickly from a health facility where they gave birth to seek more advanced care, particularly from PHCCs or health posts that provide basic emergency obstetric and newborn care only.

In this study, mothers who were accompanied by their parents, siblings or husband only were associated with longer length of stay, as compared with those who were accompanied by parents-in-laws or siblings-in-laws. This may be due to pressure from their family-in-laws to be discharged early to work at home or to show the newborn to the family at home since $60 \%$ of mothers lived with their family-in-laws in the current study. Mother-in-laws are influential in providing advice, care and support during pregnancy and after delivery. ${ }^{29}{ }^{30}$ Mothers who had more support from FCHV were more likely to stay for a shorter length in this study. FCHV might provide more support for mothers who stay for a shorter length at a health facility and return home earlier, although this study did not have data to verify this hypothesis. 
Table 3 The length of stay by reasons the mothers discharge at the timing $(n=350)$

\begin{tabular}{|c|c|c|c|c|c|c|}
\hline & & & & $\begin{array}{l}<24 \text { hours } \\
\text { stay }\end{array}$ & $\begin{array}{l}\geq 24 \text { hours } \\
\text { stay }\end{array}$ & $\begin{array}{l}\text { Length of } \\
\text { stay (hours) }\end{array}$ \\
\hline Category 1 & $\mathbf{N}$ & Category 2 & $\mathbf{N}$ & $(n=216)$ & $(n=134)$ & Median \\
\hline $\begin{array}{l}\text { Health staff's } \\
\text { suggestion }\end{array}$ & 129 & The health personnel permitted discharge & 129 & 65 & 64 & 23 \\
\hline \multirow[t]{3}{*}{ Physical condition } & 270 & A mother had a complication & 38 & 3 & 35 & 48 \\
\hline & & A newborn baby had a complication & 8 & 3 & 5 & 48 \\
\hline & & Delivery was normal & 224 & 149 & 75 & 15 \\
\hline \multirow{3}{*}{$\begin{array}{l}\text { Access to a health } \\
\text { facility }\end{array}$} & 94 & The health facility is far from the house & 16 & 14 & 2 & 11.5 \\
\hline & & The health facility is nearby the house & 59 & 47 & 12 & 12 \\
\hline & & Transportation availability & 19 & 19 & 0 & 9 \\
\hline \multirow[t]{6}{*}{$\begin{array}{l}\text { Characteristics of a } \\
\text { health facility }\end{array}$} & 90 & $\begin{array}{l}\text { Did not like the environment of the health } \\
\text { facility }\end{array}$ & 18 & 14 & 4 & 12 \\
\hline & & There was no space to stay & 22 & 14 & 8 & 12.5 \\
\hline & & The health personnel availability & 2 & 2 & 0 & 4.5 \\
\hline & & To get better care at home & 27 & 19 & 8 & 15 \\
\hline & & To take better food at home & 18 & 10 & 8 & 19 \\
\hline & & Family support was available at home & 3 & 3 & 0 & 15 \\
\hline \multirow[t]{7}{*}{ Family matters } & 54 & Family member suggested to be discharged & 9 & 6 & 3 & 12 \\
\hline & & The companion had to go back & 4 & 4 & 0 & 8.5 \\
\hline & & The mother had to do housework & 5 & 4 & 1 & 9 \\
\hline & & The mother had to do agriculture work & 3 & 2 & 1 & 21 \\
\hline & & $\begin{array}{l}\text { The mother had to take care of the older } \\
\text { children }\end{array}$ & 21 & 15 & 6 & 15 \\
\hline & & Family members were waiting at home & 3 & 3 & 0 & 8 \\
\hline & & Financial reason & 9 & 1 & 8 & 48 \\
\hline The timing of delivery & 7 & $\begin{array}{l}\text { Holiday/had an event/winter/husband was } \\
\text { sick/darkness }\end{array}$ & 7 & 5 & 2 & 9 \\
\hline
\end{tabular}

Most mothers were left for a long time at a health facility after receiving the last check-up before discharge in this study. This suggests that mothers and newborns did not always receive adequate care at proper timing, even if they stayed for more than the recommended 24 hours. According to the WHO Safe Childbirth Checklist, assessing mothers and newborns at the time of discharge is important to confirm that they are healthy enough to discharge. ${ }^{31}$ This risk assessment before discharge from health facilities plays a vital role in reducing readmission and avoiding a delay in detecting complications in mothers and newborns. ${ }^{32}$ Particularly in settings where returning to a health facility after discharge is uncommon, assessment before discharge is important. What matters is not how long they stayed, but whether they receive adequate care during their prolonged stay at a health facility and whether they can safely return to start their life at home. Further studies should be conducted to measure the quality of care during check-ups at health facilities, in addition to the timing of the check-ups.

\section{Limitations}

First, this was a cross-sectional study, and it did not examine causal inference. For example, those who stayed longer might be more likely to be diagnosed with some types of complications. However, this study provided detailed information on the option of the length of stay at a health facility and factors behind the choice, which reflects both the current level of health systems and sociodemographic conditions among mothers and their families. Second, this study was based on mothers' selfreports. Over-reporting or under-reporting could occur, particularly regarding variables that quantified their experiences and the conditions of childbirth, such as the length of stay at a health facility, the components of PNC and the child's weight of birth. Part of possible imprecise reporting might be due to a social desirability bias. The time from childbirth varies among mothers at the time of interview, and this might have affected their reports, too. Nonetheless, as this study highlighted it, health facilities might not have a reliable record on the timing of discharge owing to missing the required check-up at the 
timing of discharge. Self-reporting, however, may be the only method to measure the timing of discharge in this study area. The response rate was almost $100 \%$, which minimised the chance of selection bias. Incorporating the time of providing care in medical records should be promoted to improve the quality of medical records, and future studies should assess such records for objective measurements. Third, mothers were recruited only at public facilities, and this study did not include mothers who gave birth at private facilities. Almost $80 \%$ of facility deliveries were made at public health facilities in the province where Dhading District was located in 2016. ${ }^{20}$ Future studies should include all types of health facilities within a target area. Fourth, some mothers were excluded from this study owing to geographical access during the data collection and incomplete medical records. This can limit the generalisability of the findings. Nevertheless, this study contacted mothers by visiting their community, not by meeting them when they returned to health facilities only. This could minimise the attrition of mothers listed in the medical records. Fifth, the negative binomial regression model did not adjust for multiple comparisons. The model had 17 independent variables to test the statistical significance of each independent variable with the length of stay. Each of the significance testing involves type I error; that is, there might have an elevated possibility of committing type I error of at least one of these variables. However, this model tested if each independent variable was statistically significant and did not examine the joint significance of two or more independent variables were collectively significant, which could justify not to adjust for multiple comparisons.

\section{CONCLUSIONS}

In this study, more than half of mothers were discharged from a health facility within 24 hours after childbirth, and about two-thirds did not receive the last check-up within 3 hours before discharge in Dhading, Nepal. Multiple factors, such as mothers' conditions, facility characteristics and external support, were associated with the length of stay after childbirth. Even if mothers stayed for a long time, they might not necessarily receive high-quality care. The findings of this study call for enforcing the guidelines on the minimum length of stay after childbirth and proper assessment before discharge, in addition to improving the facility environment to enable longer stay. The level of understanding among family members should be enhanced by citing the importance of mothers and newborns staying long enough at a health facility, particularly among family members who accompany mothers and newborns, facilitate transportation and decide on household task allocation.

Acknowledgements The authors would like to express our sincere gratitude to mothers who participated in this study and family members of them. We are thankful to the hospital, the primary healthcare centre and the health posts' staff, local authorities and local people who greatly assisted our research. We are grateful to our counterparts, the members of Green Tara Nepal and research assistants for assistance with the data collection. We would like to thank Japan Medical Association and Association of Medical Doctors of Asia for supporting this research. We would like to thank Editage (www.editage.com) for English language editing.

Contributors SI conceived and designed the study. She also collected, analysed the data and wrote the first draft of the manuscript. AS supervised designing, analyses, data interpretation of this study and critically edited the manuscript. RS contributed to conducting the fieldwork. MJ obtained the funding, supervised the study and critically edited the manuscript. All authors read and approved the final draft.

Funding This work was supported through Japan Medical Association and Association of Medical Doctors of Asia. Grant number 1502000003's.

Competing interests None declared.

Patient consent for publication Not required.

Ethics approval Ethical approval was obtained from the Research Ethics Committee of the Graduate School of Medicine, the University of Tokyo (12025) and the Nepal Health Research Council (35S/2018).

Provenance and peer review Not commissioned; externally peer reviewed.

Data availability statement Data are available on reasonable request. Data are available by emailing the corresponding author.

Supplemental material This content has been supplied by the author(s). It has not been vetted by BMJ Publishing Group Limited (BMJ) and may not have been peer-reviewed. Any opinions or recommendations discussed are solely those of the author(s) and are not endorsed by BMJ. BMJ disclaims all liability and responsibility arising from any reliance placed on the content. Where the content includes any translated material, BMJ does not warrant the accuracy and reliability of the translations (including but not limited to local regulations, clinical guidelines, terminology, drug names and drug dosages), and is not responsible for any error and/or omissions arising from translation and adaptation or otherwise.

Open access This is an open access article distributed in accordance with the Creative Commons Attribution Non Commercial (CC BY-NC 4.0) license, which permits others to distribute, remix, adapt, build upon this work non-commercially, and license their derivative works on different terms, provided the original work is properly cited, appropriate credit is given, any changes made indicated, and the use is non-commercial. See: http://creativecommons.org/licenses/by-nc/4.0/.

ORCID iDs

Subaru lkeda http://orcid.org/0000-0002-0408-8658

Akira Shibanuma http://orcid.org/0000-0003-2058-1722

Masamine Jimba http://orcid.org/0000-0001-5659-3237

\section{REFERENCES}

1 WHO. WHO recommendations on maternal health: guidelines approved by the WHO guidelines review Committee. Geneva, Switzerland: WHO, 2017.

2 UNICEF. The state of the world's children 2019. New York, USA: UNICEF, 2019.

3 WHO. WHO recommendations on postnatal care of the mother and newborn. Geneva, Switzerland: WHO, 2013.

4 Campbell OMR, Cegolon L, Macleod D, et al. Length of stay after childbirth in 92 countries and associated factors in 30 low- and middle-income countries: compilation of reported data and a crosssectional analysis from nationally representative surveys. PLoS Med 2016;13:e1001972.

5 Malkin JD, Garber S, Broder MS, et al. Infant mortality and early postpartum discharge. Obstet Gynecol 2000;96:183-8.

6 Braveman P, Egerter S, Pearl M. Early discharge of newborns and mothers: a critical review of the literature. Pediatrics 1995;96:716-26.

7 Brown S, Lumley J, Small R. Early obstetric discharge: does it make a difference to health outcomes? Paediatr Perinat Epidemiol 1998;12:49-71.

8 Metcalfe A, Mathai M, Liu S, et al. Proportion of neonatal readmission attributed to length of stay for childbirth: a population-based cohort study. BMJ Open 2016;6:e012007.

9 Liu S, Heaman M, Kramer MS, et al. Length of hospital stay, obstetric conditions at childbirth, and maternal readmission: a populationbased cohort study. Am J Obstet Gynecol 2002;187:681-7.

10 Harron K, Gilbert R, Cromwell D, et al. Newborn length of stay and risk of readmission. Paediatr Perinat Epidemiol 2017;31:221-32.

11 Nipte D, Dhayarkar S, Pawar S, et al. Determinants of early discharge of mothers from hospitals after delivery in Beed block of Beed 
district, Maharashtra, India 2014. Clinical Epidemiology and Global Health 2015:3:S26-33.

12 McMahon SA, Mohan D, LeFevre AE, et al. "You should go so that others can come"; the role of facilities in determining an early departure after childbirth in Morogoro Region, Tanzania. BMC Pregnancy Childbirth 2015;15:328.

13 Milambo JPM, Cho K, Okwundu C, et al. Newborn follow-up after discharge from a tertiary care hospital in the Western Cape region of South Africa: a prospective observational cohort study. Glob Health Res Policy 2018;3:2

14 Izudi J, Akwang GD, Amongin D. Early postnatal care use by postpartum mothers in Mundri East County, South Sudan. BMC Health Serv Res 2017;17:442.

15 Berhe A, Bayray A, Berhe Y, et al. Determinants of postnatal care utilization in Tigray, Northern Ethiopia: a community based crosssectional study. PLoS One 2019;14:e221161.

16 Langlois Étienne V, Miszkurka M, Zunzunegui MV, et al. Inequities in postnatal care in low- and middle-income countries: a systematic review and meta-analysis. Bull World Health Organ 2015;93:259-70.

17 Winch PJ, Alam MA, Akther A, et al. Local understandings of vulnerability and protection during the neonatal period in Sylhet district, Bangladesh: a qualitative study. Lancet 2005;366:478-85.

18 Kearns A, Onda S, Caglia J. Postnatal care in Nepal: components of care, implementation challenges, and success factors. Massachusetts, USA: the maternal health Task force and development of global health and population at the Harvard school of public health, HPR/WHO, ICS Integrare 2014.

19 Ministry of Health Nepal. Annual report department of health services 2015/2016. Kathmandu, Nepal: Ministry of Health Nepal, 2016.

20 Ministry of Health Nepal, New ERA Nepal, ICF. Nepal demographic and health survey 2016. Kathmandu, Nepal: Ministry of Health Nepal, New ERA Nepal, ICF, 2017.
21 UNICEF country profiles. Maternal and newborn health disparities Nepal [online], 2016. Available: https://data.unicef.org/wp-content/ uploads/country_profiles/Nepal/country\%20profile_NPL.pdf

22 Perneger TV. What's wrong with Bonferroni adjustments. BMJ 1998;316:1236-8.

23 Bender R, Lange S. Adjusting for multiple testing - when and how? $J$ Clin Epidemiol 2001;54:343-9.

24 Srivastava A, Avan BI, Rajbangshi P, et al. Determinants of women's satisfaction with maternal health care: a review of literature from developing countries. BMC Pregnancy Childbirth 2015;15:97.

25 Central Bureau of Statistics. National population and housing census 2011. Dhading. Nepal: Government of Nepal National Planning Commission Secretariat, 2014.

26 Central Bureau of Statistics. Population monograph of Nepal. government of Nepal national planning Commission Secretariat. Nepal: Central Bureau of Statistics, 2014

27 Upadhyay P, Liabsuetrakul T, Shrestha AB, et al. Influence of family members on utilization of maternal health care services among teen and adult pregnant women in Kathmandu, Nepal: a cross sectional study. Reprod Health 2014;11:92.

28 Simkhada B, Teijlingen ERvan, Porter M, et al. Factors affecting the utilization of antenatal care in developing countries: systematic review of the literature. J Adv Nurs 2008:61:244-60.

29 Paudel M, Javanparast S, Newman L, et al. Health system barriers influencing perinatal survival in mountain villages of Nepal: implications for future policies and practices. J Health Popul Nutr 2018;37:16-19.

30 Thapa DK, Niehof A. Women's autonomy and husbands' involvement in maternal health care in Nepal. Soc Sci Med 2013;93:1-10.

31 WHO. Who safe childbirth checklist implementation guide: improving the quality of facility-based delivery for mothers and newborns. Geneva, Switzerland: WHO, 2015.

32 WHO. Who recommendations: intrapartum care for a positive childbirth experience. Geneva, Switzerland: WHO, 2018. 